\title{
Grupo de apoio para pais de crianças com implante coclear
}

\section{Support group for parents of children with cochlear implant}

\section{Grupo de apoyo para padres de niños con implante coclear}

\author{
Fernanda Pádua Rezende* \\ Hospital de Reabilitação de Anomalias Craniofaciais - HCRAC/USP, Bauru, São \\ Paulo, Brasil
}

\section{Marina Morettin**}

Hospital de Reabilitação de Anomalias Crânio Faciais - HCRAC/USP, Bauru, São Paulo, Brasil

\section{Midori Otake Yamada***}

Hospital de Reabilitação de Anomalias Crânio Faciais - HCRAC/USP, Bauru, São Paulo, Brasil

\begin{abstract}
RESUMO
O objetivo deste artigo foi apresentar um levantamento bibliográfico sobre grupos de apoio para pais de crianças com implante coclear e discutir os resultados encontrados, buscando evidenciar a importância e os benefícios da utilização dessa ferramenta de auxílio aos pais e cuidadores. Entre os meses de setembro de 2013 e março de 2014 foi realizada a busca de estudos sobre "Grupo de Apoio para pais de crianças com deficiência auditiva, usuárias de implante coclear" nas bases de dados eletrônicas Pubmed, Scielo e Lilacs, utilizando a combinação de descritores selecionados previamente. Após este levantamento, 15 estudos foram selecionados para análise e discussão. Verificou-se a escassez da literatura sobre o tema. No entanto, foi possível constatar que a realização de grupos de apoio com a família de crianças ou pacientes com determinadas deficiências, ou outras condições de saúde, é uma ferramenta de extrema importância, pois permite aos pais enfrentarem as situações complexas com mais segurança, promovendo trocas de experiências, reflexão, alívio do estresse, além de amenizar a ansiedade entre os familiares. Portanto, os estudos analisados indicam a necessidade de algum tipo de suporte aos pais no enfrentamento da deficiência auditiva de seus filhos, de outras deficiências ou condições de saúde, sendo uma ferramenta de suporte emocional fundamental.
\end{abstract}

Palavras-chave: apoiadores, pais, implante coclear.

\section{ABSTRACT}

The objective was to present a literature review about support groups for parents of children with cochlear implants and discuss the results in order to enhance the importance and benefits of using this tool to help parents and caregivers. Between the months of September 2013 and March 2014 a 
search was performed for studies on "Support Group for parents of hearing impaired children with cochlear implants" in electronic databases Pubmed, Scielo and Lilacs, using the combination of descriptors previously selected. After this survey, 15 studies were selected for analysis and discussion. A literature search of the subject indicated a lack there of. However it was found that the performance of support groups with the family of children or patients with certain disabilities or other health conditions is an extremely important tool because it allows parents face complex situations safer, promotes exchange of experiences among family, reflection, stress relief, and decrease anxiety. Therefore, the studies analyzed indicate the need for some kind of support to parents in coping with hearing loss of their children, other disabilities or health conditions, as a fundamental emotional support tool.

Keywords: support groups, parents, cochlear implant.

\section{RESUMEN}

El presente documento tiene por objetivo recabar la literatura existente sobre la importancia y los beneficios de los grupos de apoyo para padres y cuidadores de niños con implantes cocleares en el uso de esta herramienta. Para lograr este cometido, entre septiembre de 2013 y marzo de 2014 se buscaron estudios en las librerías electrónicas de prestigiosas entidades como Pubmed, Scielo y Lilacs, a través de descriptores previamente seleccionados. Aun cuando la literatura asociada a este tema es escasa, se seleccionaron 15 documentos que fueron analizados y discutidos, deduciendo que la ayuda prestada por los grupos de apoyo a las personas cercanas a los niños con alguna discapacidad física o de salud es vital, porque les permite actuar con mayor seguridad en situaciones complejas, promueve el intercambio de experiencia, da espacio a la reflexión, alivia el estrés y la ansiedad. Por lo tanto, de los estudios analizados se concluye que los grupos de apoyo son una herramienta fundamental para el apoyo emocional de padres y cercanos a los niños con discapacidades o problemas de salud, en especial para hacer frente a la pérdida de audición de los menores.

Palabras clave: grupos de apoyo, los padres, implante coclear.

\section{Introdução}

O ser humano, de acordo com Farah (2009), como ser social, está inserido em diversos grupos ao longo de sua vida com os quais adquire valores que farão parte de sua personalidade. A formação de grupos acontece de forma natural na vida do indivíduo, por meio de afinidades, de gostos que se parecem, estilos e práticas comportamentais em comum.

Os grupos, como formas naturais do homem relacionar-se, também podem ser formados de outras maneiras, em outros contextos que não seja aquele rotineiro, como por exemplo, em serviços de saúde. Apesar disso, a possibilidade de intervenção grupal tem sido pouco explorada e aproveitada nestas situações e, segundo Bleger (1998), onde há pessoas, existe a possibilidade de formação de grupos. Desta forma é interessante que o profissional vá até estes indivíduos com a 
proposta de intervenção grupal e não espere que estes o procure para atendimento, pois nem sempre este atendimento irá abranger tudo o que pode ser contemplado de forma coletiva (Bleger, 1998).

Isto por que, um atendimento coletivo poderá promover a identificação, parceria de trabalho, troca de papeis e melhor integração entre os participantes, desencadeando, a partir deste contato com as outras pessoas, um processo de aprendizagem. Esta aprendizagem se dá, na medida em que há questionamentos acerca de si mesmo e dos outros, abrindo a possibilidade de um olhar mais amplo sobre a realidade vivida, dando condições para as mudanças necessárias na vida destas pessoas (Pichon-Rivière, 1998).

De acordo com as considerações levantadas nos trabalhos de PichonRivière (1998) e Bleger (1998), e a partir da observação de que as mães de crianças com deficiência auditiva formavam um grupo espontâneo ao se encontrarem na sala de espera durante o acompanhamento dos seus filhos na rotina do Centro de Pesquisas Audiológicas (CPA) do Hospital de Reabilitação de Anomalias Craniofaciais (HRAC), Universidade de São Paulo (USP), campus Bauru, responsável pelo programa de implante coclear (PIC), um grupo de apoio aos pais de crianças usuárias de implante coclear (IC) foi formado no serviço.

Ribeiro, Yamada e Tavano (2007) referiram em seu estudo que, de certa forma este momento na sala de espera proporcionava um espaço de ajuda mútua, de aprendizagem e troca de experiências entre as famílias. Porém, segundo as autoras, este encontro espontâneo também desencadeava incômodo, insegurança e ansiedade em algumas famílias, visto que a demanda de informações nem sempre se aplica para todos os casos, levando à equipe do serviço à reflexão de que estes sentimentos poderiam ser aliviados com a organização de um espaço propício e adequado para a reunião deste grupo.

Segundo Pichon-Rivière (1998) o que caracteriza o processo grupal é - constante movimento de estruturação, desestruturação e reestruturação, envolvendo o desejo de mudança, o contato com o novo, além de ansiedade e medos que podem gerar a resistência para a transformação. Com a análise sistemática das contradições, por exemplo, entre velho e novo, necessidade e satisfação, sujeito e grupo, os processos grupais vão se desenvolvendo rumo a aprendizagem de novos pensamentos, desejos e anseios (Pereira, 2013), fato que foi observado por Ribeiro, Yamada e Tavano (2007) com as mães em sala de espera.

Outro aspecto relevante, que vale destacar, é a técnica de "grupos operativos" na concepção de Pichon-Rivière (1998). Para o autor esta técnica, envolve, de forma não diretiva, os participantes em um ambiente de investigação ativa, ou seja, cabe ao condutor do grupo a tarefa de facilitar a comunicação entre os participantes, 
proporcionando a ideia de operativo (Pereira, 2013). O sujeito tornase ativo e central no processo de construção de novas experiências e conhecimentos, não é mero recebedor de informação, mas agente da própria mudança, que contribui para a transformação do outro (Pereira, 2013).

Dessa forma, teve início em 2004, o "Grupo de Apoio Psicossocial para pais e acompanhantes de pacientes com Implante Coclear" (Ribeiro, Yamada e Silva, 2005). A intenção da formação desse grupo foi fornecer um espaço para as famílias compartilharem suas vivências, sejam elas em relação à deficiência auditiva de seus filhos, experiências com o uso do IC, dificuldades e conquistas no processo de habilitação da criança, assim como temáticas relacionadas às suas vidas, sendo este um momento de apoio para os pais expressarem seus pensamentos e sentimentos. Os profissionais que atuam na dinâmica do encontro são assistentes sociais e psicólogos constituindo, assim, um grupo de apoio psicossocial com periodicidade de duas vezes por semana e com duração de uma hora (Ribeiro, Yamada, Silva, 2005).

Inspirado pelo trabalho de grupo realizado no CPA com os pais de crianças com IC, no qual a autora participa como prática do Programa de Residência Multiprofissional em Saúde Auditiva do HRAC/USP, este estudo teve como objetivo apresentar um levantamento bibliográfico sobre grupos de apoio para pais de crianças com implante coclear, a fim de verificar se há publicações nacionais e internacionais sobre o assunto e discutir os resultados encontrados, buscando evidenciar a importância e os benefícios da utilização dessa ferramenta de auxílio, como suporte emocional, para enfrentamento da deficiência auditiva aos pais de crianças com implante coclear.

\section{Método}

Consistiu em uma revisão crítica da literatura sobre grupos de apoio, especificamente, direcionados para pais de crianças que usam IC.

A revisão da literatura foi realizada nas bases de dados eletrônicas Pubmed, Scielo e Lilacs. Os descritores utilizados em língua portuguesa foram: perda auditiva, implante coclear, criança, pais, família, psicologia, prática de grupo, aconselhamento, psicoterapia de grupo e grupos de apoio. Na língua inglesa foram usados: self-help groups, hearing loss, child, parents, cochlear implant, psychology, group practice, counseling psychotherapy, group, family e self-help groups. Outra estratégia utilizada para seleção dos estudos foi a revisão das referências citadas nos artigos selecionados para inclusão na revisão de literatura.

O critério de inclusão dos artigos foi definido de acordo com a pertinência com o tema "grupo de apoio", focando estudos 
relacionados à deficiência auditiva e uso de implante coclear. No entanto, foram também incluídos estudos que abordaram o tema principal deste levantamento bibliográfico relacionados com outras deficiências e problemas de saúde, a fim de permitir a reflexão dos trabalhos que envolveram grupos de apoio em outros contextos e que podem contribuir com diferentes informações sobre esta temática.

$\mathrm{O}$ ano de publicação dos estudos não foi restrito, dando preferência para seleção de estudos em português e inglês.

Após seleção dos estudos, os mesmos foram lidos e analisados de acordo com seus resultados, sendo apresentados os resultados em uma tabela (Tabela 1) que sintetizou as informações relevantes de cada artigo (título, autores, ano, revista e um resumo do tema central do estudo).

\section{Resultados e discussão}

A partir do levantamento realizado com os descritores definidos previamente nas bases de dados, encontrou-se 158 artigos publicados ( 9 no Lilacs, 146 no Pubmed e 5 no Scielo). Após a análise dos estudos, seguindo o critério de pertinência com o tema, e da análise das referências bibliográficas dos artigos selecionados, ao final foram incluídos na revisão bibliográfica sete (07) estudos que abordavam a realização de grupos de pais com crianças com deficiência auditiva, outras deficiências ou problemas de saúde. No entanto, a fim de complementar as discussões em relação à importância do suporte psicossocial aos pais, cinco (05) artigos foram inseridos, além de outros três (03) que revisaram os benefícios da psicoterapia de grupo, fortalecendo as discussões levantadas nesses estudos. Assim, ao final, 15 estudos foram incluídos nesta revisão bibliográfica.

Dos 15 estudos selecionados, o estudo mais antigo foi publicado no ano de 1998 e o mais recente em 2012. Em relação aos temas centrais abordados nos estudos selecionados (Tabela 1) verificou-se que apenas três tratam a respeito de grupo de pais de crianças com deficiência auditiva, sendo que destes, um está relacionado com pais de crianças usuárias de IC. Quatro estudos estavam relacionados com outras deficiências e problemas de saúde listados a seguir: grupo de apoio para pais de crianças com deficiência visual, grupo de apoio para familiares de pacientes com anorexia e bulimia, grupo de apoio para pais de crianças internadas em UTI neonatal e grupo de apoio para familiares de crianças com deficiências em geral. Cinco artigos mostraram a necessidade de ser oferecido pela equipe de saúde um suporte psicossocial aos pais, a fim de permitir o enfrentamento do diagnóstico e tratamento da deficiência auditiva de seus filhos, além 
de outras três revisões de literatura tratarem sobre os benefícios da psicoterapia de grupo em geral.

\section{Tabela 1. Artigos selecionados}

\begin{tabular}{|c|c|c|c|c|}
\hline Título & Autor & Ano & Revista & Tema central \\
\hline $\begin{array}{l}\text { 1) Intervention } \\
\text { with groups of } \\
\text { parents of } \\
\text { preschool } \\
\text { children with } \\
\text { hearing loss }\end{array}$ & $\begin{array}{l}\text { Rena, F. \& } \\
\text { Mirian, P. }\end{array}$ & 1998 & Volta Review & $\begin{array}{l}\text { Grupo de pais de } \\
\text { crianças com } \\
\text { deficiência } \\
\text { auditiva } \\
\text { moderada/profun } \\
\text { da. }\end{array}$ \\
\hline $\begin{array}{l}\text { 2) The need for } \\
\text { psychosocial } \\
\text { support in parents } \\
\text { with cochlear } \\
\text { implanted children }\end{array}$ & $\begin{array}{l}\text { Spanh C., } \\
\text { Richter, B., } \\
\text { Zschocke, I., } \\
\text { Löhle, E. \& } \\
\text { Wirsching M }\end{array}$ & 2001 & $\begin{array}{l}\text { International } \\
\text { Journal of } \\
\text { Pediatric } \\
\text { Otorhinolaryn } \\
\text { gology }\end{array}$ & $\begin{array}{l}\text { Necessidade de } \\
\text { suporte para pais } \\
\text { de crianças com } \\
\text { IC }\end{array}$ \\
\hline $\begin{array}{l}\text { 3) Psicoterapia de } \\
\text { grupo e } \\
\text { considerações } \\
\text { sobre o paciente }\end{array}$ & $\begin{array}{l}\text { Bochelli, L. } \\
\text { P. C. \& } \\
\text { Santos, M. } \\
\text { A. }\end{array}$ & 2002 & $\begin{array}{l}\text { Rev. Latino- } \\
\text { am } \\
\text { Enfermagem }\end{array}$ & $\begin{array}{l}\text { Benefícios da } \\
\text { psicoterapia de } \\
\text { grupo ao } \\
\text { paciente }\end{array}$ \\
\hline
\end{tabular}

agente da própria mudança

\begin{tabular}{|c|c|c|c|c|}
\hline $\begin{array}{l}\text { 4) A deficiência } \\
\text { auditiva e a familia: } \\
\text { sentimentos e } \\
\text { expectativas de um } \\
\text { grupo de pais de } \\
\text { crianças com } \\
\text { deficiência da } \\
\text { audição }\end{array}$ & $\begin{array}{l}\text { Boscolo, C. } \\
\text { C. \& Santos, } \\
\text { T. M. M. }\end{array}$ & 2005 & $\begin{array}{l}\text { Distúrbios da } \\
\text { Comunicação }\end{array}$ & $\begin{array}{l}\text { Necessidade de } \\
\text { suporte/compart } \\
\text { lhar sentimentos } \\
\text { dos pais de } \\
\text { crianças com } \\
\text { deficiência } \\
\text { auditiva }\end{array}$ \\
\hline $\begin{array}{l}\text { 5) Grupo de } \\
\text { acompanhantes de } \\
\text { pacientes com } \\
\text { implante coclear: } \\
\text { uma ação } \\
\text { interdisciplinar da } \\
\text { psicologia e do } \\
\text { serviço social }\end{array}$ & $\begin{array}{l}\text { Ribeiro, S. F. } \\
\text { R. Yamada, } \\
\text { M. O. \& } \\
\text { Silva, C. }\end{array}$ & 2005 & $\begin{array}{l}\text { Revista da } \\
\text { SPAGESP }\end{array}$ & $\begin{array}{l}\text { Grupo de apoio } \\
\text { psicossocial aos } \\
\text { pais de crianças } \\
\text { com IC }\end{array}$ \\
\hline $\begin{array}{l}\text { 6) O Paciente na } \\
\text { psicoterapia de } \\
\text { grupo }\end{array}$ & $\begin{array}{l}\text { Bochelli, L. } \\
\text { P. C. \& } \\
\text { Santos, M. } \\
\text { A. }\end{array}$ & 2005 & $\begin{array}{l}\text { Rev. Latino- } \\
\text { am } \\
\text { Enfermagem }\end{array}$ & $\begin{array}{l}\text { Benefícios da } \\
\text { psicoterapia de } \\
\text { grupo ao } \\
\text { paciente }\end{array}$ \\
\hline $\begin{array}{l}\text { 7) Vivência de } \\
\text { mães de crianças } \\
\text { com deficiência } \\
\text { auditiva em sala de } \\
\text { espera }\end{array}$ & $\begin{array}{l}\text { Ribeiro, S. F. } \\
\text { R. Yamada, } \\
\text { M. O. \& } \\
\text { Tavano, L. } \\
\text { D. }\end{array}$ & 2007 & $\begin{array}{l}\text { Psicologia em } \\
\text { Revista }\end{array}$ & $\begin{array}{l}\text { Necessidade de } \\
\text { suporte para } \\
\text { mães que tem } \\
\text { filhos com } \\
\text { deficiência } \\
\text { auditiva }\end{array}$ \\
\hline
\end{tabular}


8) Mães de crianças com deficiência visual: percepções, conduta e contribuição do atendimento em grupo

9) Psicoterapia de grupo: reflexões sobre as mudanças no contato entre os membros do grupo durante o processo terapêutico.

10) Experiência de Grupo de Pais em uma Unidade de Terapia Intensiva Neonatal

11) Fatores terapêuticos em um grupo de apoio multifamiliar no tratamento da anorexia e bulimia

12) Grupo de apoio psicológico: vivenciando a maternidade de um filho especial 13) Family spports and resources for parents of children who are deaf or hard of hearing

14) Psychological aspects when counseling families who have children with cochlear implants 15) Grupoterapia e deficiência auditiva infantil: trabalhando com mães e crianças
Nobre, M. I. 2008

R. S.

Montilha, R.

C. I. \&

Temporini,

E. R.

Farah, A.B.Z. 2009

Revista IGT na

Ferreira, J. 2009 PEDIATRIA

C. O. A.

Sakita, N. K.

\& Ceccon $\mathrm{M}$.

E. J. R.

Souza, L. V., 2010 Rev.

Santos, M.

A., Moura, F.

E. G. A.,

Campos-

Brustelo, T.

N. \& Savioli,

C. M. G.

Kunst, G. C.

E. Machado,

M. L. R. L. \&

Ribeiro, $M$.

M. S. M.

Jackson, C. w.

2011

2010

Fadda, S.

2011

The Journal of

Silva, N. M. 2012 Paiva, F. C. \& Silveira, A. A. B. Rede

Revista

brasileira de crescimento e desenvolvime nto humano

SPAGESP

Revista

Conhecimento

Online

American

Annals of the Deaf Maternal-Fetal and Neonatal Medicine

Grupo de apoio para mães de crianças com deficiência visual

Benefícios da psicoterapia de grupo

\author{
Gripo de apoio \\ para pais de \\ crianças na UTI \\ neonatal \\ Grupo de apoio \\ para familiares \\ de pacientes com \\ anorexia e \\ bulimia
}

\section{Grupo de apoio psicológico para mães de crianças com deficiência}

Necessidade de suporte para a familia de crianças com deficiência auditiva Necessidade de suporte para familias com filhos que usam IC

Revista da Grupo de apoio SPAGESP para mães de crianças com deficiência auditiva

De acordo com o conteúdo analisado na literatura médica levantada, ter um filho com algum tipo de deficiência gera sofrimento familiar. A deficiência auditiva não é diferente, pois os pais quando se deparam com a situação de ter um filho com deficiência auditiva precisam se 
reestruturar e ao mesmo tempo estar envolvidos com o processo de tratamento da criança. Isto por que, o sucesso da intervenção da deficiência auditiva em crianças dependerá da participação efetiva da família durante os primeiros anos após o diagnóstico. É a família quem irá, após ser orientada pela equipe de profissionais que realizaram o diagnóstico da perda auditiva, tomar a decisão sobre a melhor opção de tratamento do seu filho, que pode fazer uso do aparelho de amplificação sonora individual (AASI) ou implante coclear (IC), dependendo das características da perda auditiva.

Dessa forma, Jackson (2011) enfatiza que a família com suporte tem maiores condições emocionais para lidar com a deficiência auditiva dos seus filhos, sendo que este suporte pode vir de várias fontes, como da região em que vivem (suporte social), familiares próximos, amigos, outros pais e profissionais que atuam na reabilitação/habilitação da criança. Segundo o autor, um dos recursos mais importantes citados pelos pais de crianças com deficiência auditiva em seu estudo foi a discussão com outros pais de crianças com deficiência auditiva, pois permite que identifiquem situações comuns entre eles, auxiliando na troca de informações. Dessa forma, esses resultados valorizam a necessidade de promover entre essas famílias situações que permitam a interação pessoal com outras pessoas que tenham vivido uma experiência semelhante.

Esta necessidade de enfrentar a situação gerada pela deficiência auditiva conversando com pessoas que vivenciam algo semelhante também foi constatada em outros estudos. Fadda (2011), em um levantamento bibliográfico sobre a atuação do psicólogo durante o processo de diagnóstico da deficiência auditiva até a reabilitação com o implante coclear em crianças, teve como uma de suas constatações que os pais destas crianças buscavam conhecer outros pais que tinham filhos na mesma situação, para que assim pudessem identificar sentimentos semelhantes, buscando estratégia de enfrentamento frente à fase de diagnóstico.

Boscolo e Santos (2005) ao entrevistarem pais de filhos com deficiência auditiva usuários de AASI perceberam em seus discursos o interesse dos pais de procurarem outras famílias na mesma situação para compartilharem conhecimentos a fim de minimizar o sofrimento gerado pelo diagnóstico e tratamento da perda auditiva, além de poderem trocar informações e experiências vividas por eles no dia a dia com seus filhos.

Ribeiro, Yamada e Tavano (2007) analisaram depoimentos de mães de crianças em fase de diagnóstico e tratamento da deficiência auditiva e identificaram em suas falas esta mesma necessidade de troca de informações, compartilhar sentimentos, ideias e experiências, como forma de amenizar o sofrimento gerado pela condição de se ter um filho com deficiência auditiva. 
Assim, nota-se que há diversos sentimentos em torno da família que precisam ser trabalhados e ouvidos. Tais sentimentos permeiam todo o processo de intervenção da criança, desde o diagnóstico audiológico até a reabilitação/habilitação auditiva, que envolve a adaptação de dispositivos eletrônicos (AASI e/ou IC) e a realização da terapia fonoaudiológica para desenvolvimento das habilidades de audição e de linguagem da criança. Spanh, Richter, Zschocke, Löhle e Wirsching (2001) e Fadda (2011) enfatizam a importância da família ser acompanhada por profissionais da área durante todo este processo, a fim de identificar os pais que necessitam do suporte psicossocial para enfrentarem as suas condições psíquicas, como altos níveis de estresse, ansiedade e depressão.

Dessa forma, é observada na literatura a necessidade por parte de pais de crianças com deficiência auditiva que fazem ou não uso de IC de algum tipo de suporte, que ofereça orientação, aconselhamento, troca de informação, sendo, portanto, uma maneira de amparo, acolhimento e ajuda.

Uma opção de suporte que pode ser oferecido à família de crianças usuárias de AASI e/ou IC é a psicoterapia de grupo. Este tipo de intervenção, que pode ser oferecida no próprio serviço que acompanha a criança e família, oferece a elas ferramentas para enfrentarem determinada situação problemática da vida ou modificar certos comportamentos que trazem prejuízos para o desenvolvimento infantil.

Bochelli e Santos (2002) afirmam que a psicoterapia de grupo é tão eficaz quanto a terapia individual, sendo uma técnica destinada a toda e qualquer pessoa que busque sua autoconsciência e não apenas para as pessoas consideradas doentes. Assim, segundo os autores, este conhecimento de si é favorecido pelo contato interpessoal que o grupo proporciona.

Os autores ainda mencionam que os assuntos a serem trabalhados devem ser trazidos pelos integrantes do grupo, e os membros devem encontrar auxilio entre si em um espaço que proporcione a liberdade de participação. Neste ambiente, o diálogo estabelecido com os integrantes deve ter uma escuta empática, com perguntas que levem ao esclarecimento dos assuntos. Além disso, no processo grupal há troca de informações, ideias, interpretações, apoio, feedback, conselhos e sugestões de estratégias de enfrentamento e, portanto, como disseram Bochelli e Santos (2002) "o apoio mútuo consolida a união de grupo em torno de um objetivo comum" (p.388).

O profissional atuante no grupo também tem seu papel de assegurar o andamento do mesmo e favorecer as dinâmicas. Espera-se do profissional a habilidade e conhecimento para manter a cultura e funcionamento do processo grupal, além de atuar com os fatores terapêuticos, estimular a reflexão e fornecer o apoio. $O$ simples fato de estar no grupo ouvindo outras pessoas, sentindo-se aceito, pode 
funcionar como mecanismo de aprendizado interior e pode levar a estimulação de novas atitudes (Bochelli \& Santos, 2002, 2005).

Para Farah (2009) a técnica grupal é uma ferramenta que promove suporte, ensina e faz com que as pessoas compartilhem suas experiências de maneira singular de acordo com as relações que são estabelecidas. Dessa forma, é algo diferente de uma simples conversa, pois gera um ambiente no qual há suporte e apoio para as pessoas falarem de si mesmas, de situações desagradáveis e que geram sofrimento. Além do terapeuta participar como acolhedor, os demais participantes atuam também desta forma. Ainda segundo o autor, o maior diferencial que a psicoterapia de grupo traz é a troca de experiências, auxiliando o participante a visualizar que não se está sozinho diante daquele problema.

Sendo assim, o grupo é considerado uma técnica utilizada por profissionais na área da saúde que auxilia no apoio e suporte aos pacientes. Na literatura, encontram-se diversos trabalhos que mostram a eficácia de grupos de apoio, tanto na promoção de mudanças de comportamentos em pacientes, como no auxilio para enfrentamento de alguma situação problemática voltado para a família de pacientes. Na Tabela 2, encontram-se os tipos de grupos de apoio analisados neste estudo e quais os resultados que esta técnica proporcionou para os participantes. 
Tabela 2. Caracterização dos grupos e benefícios gerados

\section{Tipos de grupo de apoio}

Benefícios do grupo aos participantes

Para mães de crianças com • Recebem informações sobre a deficiência visual

Para familiares de pacientes com anorexia e bulimia

Para pais de crianças internadas em UTI neonatal

Para mãe de crianças com outras deficiências

Para pais de crianças com deficiência auditiva deficiência através da troca de experiência, 0 que gera aprendizado como melhores formas de lidar com a criança;

- Esclarece dúvidas;

- Sentem-se a vontade para falarem de seus sentimentos.

Gera aprendizado para lidarem com a situação, os membros do grupo oferecem este conhecimento.

Ajuda a aliviar sentimentos desagradáveis.

Oferece aprendizado para lidarem com a situação.

- Facilita a expressão de sentimentos;

- Oferece força através dos outros pais;

- Gera aprendizado;

- Ajuda a reduzir a ansiedade gerada pelo diagnóstico.

Tomando como foco o efeito de grupos de apoio para familiares Nobre, Montilha e Temporini (2008) estudaram as contribuições do grupo de apoio às mães de crianças com deficiência visual. Aplicaram um questionário com 14 mães participantes do estudo e constataram que os benefícios gerados pelo grupo por meio da troca de experiências com as outras mães foram a respeito das oportunidades em receber informações sobre a deficiência do filho e com isso aprendiam a lidar melhor com a criança no dia a dia. Além disso, as mães acreditavam que o grupo auxiliou no esclarecimento de dúvidas, a compreenderem melhor a criança e seu desenvolvimento com a deficiência visual, visto que compartilhavam com as outras mães as mesmas experiências, o que levaram-nas a exporem seus sentimentos com menos receio.

Em outro estudo a respeito das contribuições terapêuticas em um grupo de apoio para a família no tratamento da anorexia e bulimia, Souza, Santos, Moura, Campos-Brustelo e Savioli, (2010) mostram que a família sendo orientada e tendo seus sentimentos acolhidos, entendidos e trabalhados pode lidar melhor com a condição de seu parente em tratamento. Verificaram que a participação no grupo de apoio permitiu a estas famílias durante a troca de experiências com 
outras famílias aprenderem e serem capazes de eles próprios encontrarem soluções para o problema, desenvolvendo estratégias e encontrando caminhos saudáveis e úteis para cada um deles.

Esta necessidade de dividir e adquirir informações, de ter aprendizado, de ter um momento de desabafar também foi analisado no artigo de Ferreira, Sakita e Ceccon (2009). Os autores perceberam que o grupo de apoio desenvolvido com pais de crianças internadas em UTI neonatal auxiliou no alívio dos sentimentos desagradáveis que experimentavam. Através de entrevistas após a realização do grupo, os pais concluíram que as sensações/sentimentos desagradáveis geradas com a internação de seus filhos foram diminuindo, principalmente com as informações obtidas no grupo.

Kunst, Machado e Ribeiro (2010) estudaram o grupo de apoio realizado com mães de crianças com deficiências no geral e perceberam através das suas falas que a realização do grupo auxiliou as famílias, no sentido de promover a saúde por meio do aprendizado de novas formas de enfrentar a condição dos seus filhos, proporcionando uma melhora no ato de cuidar da criança.

Assim, a falta de informações a respeito da situação que vivenciam pode desencadear ansiedade no sentido de não saberem o que fazer e como fazer, tanto no caso do paciente, como da família. Qualquer acontecimento novo exige novos comportamentos que requerem aprendizagem, e, nesse sentido, o grupo também pode ter este desdobramento, como alguns estudos mostraram.

Rena e Mirian (1998) ao trabalharem com pais de crianças com perda auditiva em grupos de apoio, com a finalidade de favorecer a comunicação entre pais e filhos, verificaram que além do aprendizado com as informações que recebiam sobre como lidar com as crianças, estes também puderam expressar sentimentos, adquirir forças e novas formas de lidar com suas expectativas diante da realidade. Este mesmo resultado foi encontrado no estudo de Silva, Paiva e Silveira (2012), com grupo de apoio para mães de crianças com deficiência auditiva, que além da aprendizagem de como lidar com o filho, encontraram um espaço propício e seguro para falarem de seus sentimentos, levando a redução do sentimento de ansiedade e amenizando o sofrimento ocasionado pela deficiência auditiva de seus filhos.

Dessa maneira, de acordo com o levantamento da literatura foi possível verificar muitos benefícios da técnica grupal, especificamente aquela voltada para dar apoio aos pais e familiares de crianças que têm alguma deficiência ou estão em situação de vulnerabilidade, como no caso da criança estar internada em UTI neonatal ou passar por anorexia e bulimia. Portanto, é pelo benefício que esta técnica gera que há o grupo de apoio para pais e acompanhantes de pacientes que fazem uso de IC no PIC do CPA. Ribeiro, Yamada e Silva (2005) mostram em seu estudo que o objetivo deste trabalho é 
favorecer a troca de experiências e trabalhar os conteúdos que os participantes trazem no dia, e o profissional atua de forma pontual, auxiliando na reflexão e fornecendo orientações na medida do possível.

Atualmente, este grupo de apoio psicossocial do CPA ocorre duas vezes por semana e é oferecido aos pais de crianças que são usuárias de implante coclear. É caracterizado como um grupo aberto onde a cada encontro há a presença de novos participantes, e, sendo assim, os conteúdos emergentes são finalizados em um único encontro que proporciona a liberdade de expressão aos pais que levam as temáticas vivenciadas em relação a deficiência auditiva e uso do IC de seus filhos (Ribeiro, Yamada \& Silva, 2005).

No entanto, até o presente momento não se sabe qual é a percepção destes pais a respeito do grupo de apoio psicossocial, e se realmente o mesmo cumpre com os objetivos propostos e se segue de acordo com o que a literatura mostra. Diante desta colocação, novos estudos poderiam ser realizados para verificar os resultados do grupo de apoio psicossocial promovido no CPA, por exemplo, na perspectiva dos pais, a fim de verificar os benefícios do mesmo e adequar os objetivos diante dos resultados obtidos.

\section{Considerações finais}

O levantamento bibliográfico realizado permitiu concluir que a técnica grupal é capaz de proporcionar diversos benefícios para seus participantes. Segundo constatado pela literatura, o grupo permite a troca de experiências, o contato com novos conhecimentos, modificação de ideias, e os membros são instigados a refletirem sobre a situação problemática, conseguindo muitas vezes aliviarem o estresse, a ansiedade, os medos e angústias.

O grupo de apoio é um facilitador para as pessoas fazerem colocações sobre suas vidas, e é um momento no qual percebem semelhanças e concluem que não estão sozinhos vivenciando determinado problema. Sentem-se, portanto, amparados e renovam as forças para o enfrentamento daquilo que é gerador de sofrimento. Apesar de poucos estudos encontrados que abordam esse tema, principalmente em relação a grupo de apoio para pais de crianças com IC, a análise da literatura mostrou estudos que enfatizam a necessidade de apoio aos pais, familiares ou cuidadores de crianças com alguma deficiência, pois muitas vezes os mesmos demonstram sentirem angústias, dúvidas, medos e estresse. $O$ trabalho de grupo foi fundamentado como técnica de apoio a esta população com resultados significativos que parecem funcionar muito bem como suporte e apoio, ou seja, cumpre com este objetivo e ajuda no enfrentamento de situações diversas. 
Esta prática inserida no CPA com os pais e acompanhantes dos pacientes usuários de IC tem respaldo técnico e científico mostrando especialmente como o trabalho de grupo auxilia os participantes a lidarem com seus sentimentos, ansiedades, medos e angústias gerados pela deficiência auditiva/implante coclear e outras situações adversas. É um espaço de apoio, onde há troca de experiências e informação, e amparo psicológico e social. Como Spanh et al. (2001) mostraram, há necessidade de suporte aos pais desde o diagnóstico da deficiência auditiva e durante todo o processo que envolve o uso de IC.

Portanto, como já foi mencionado, fica a inspiração para um estudo aprofundado para investigar a percepção destes pais a respeito do grupo e se realmente na opinião deles, cumpre com estes objetivos. Também seria interessante que este tipo de prática pudesse ser inserido com mais frequência nas demais instituições de saúde, uma vez que auxilia e muito no apoio e suporte aos acompanhantes de pacientes.

\section{Referências}

Bochelli, L. P. C., \& Santos, M. A. (2002). Psicoterapia de grupo e considerações sobre o paciente como agente da própria mudança. Rev. Latino-am Enfermagem, 10(3), 383-391.

Bochelli, L. P. C., \& Santos, M. A. (2005). O paciente na psicoterapia de grupo. Rev. Latino-am Enfermagem, 10(1), 118-125.

Boscolo, C. C., \& Santos, T. M. M. (2005). A deficiência auditiva e a família: sentimentos e expectativas de um grupo de pais de crianças com deficiência da audição. Distúrbios da comunicação, 17(1), 69-75.

Bleger, J. (1998). Temas de psicologia: entrevistas e grupos. São Paulo: Martins Fontes.

Fadda, S. (2011). Psychological aspects when counseling families who have children with cochlear implants. The Journal of MaternalFetal and Neonatal Medicine, 24(1), 104-106.

Farah, A. B. Z. (2009). Psicoterapia de grupo: Reflexões sobre as mudanças no contato entre os membros do grupo durante 0 processo terapêutico. Revista IGT na Rede, 6(11), 302-328.

Ferreira, J. C. O. A., Sakita, N. K., \& Ceccon, M. E. J. R. (2009). Experiência de grupo de pais em uma unidade de terapia intensiva neonatal. Pediatria, 31(1), 20-25.

Jackson, C. W. (2011). Family spports and resources for parents of children who are deaf or hard of hearing. American annals of the deaf, 156(4), 343-362. 
Kunst, G. C. E., Machado, M. L. R. L., \& Ribeiro, M. M. S. M. (2010) Grupo de apoio psicológico: vivenciando a maternidade de um filho especial. Revista conhecimento online, 2(2), 1-14.

Nobre, M. I. R. S., Montilha, R. C. I., \& Temporini, E. R. (2008) Mães de criança com deficiência visual: percepções, conduta e contribuição do atendimento em grupo. Revista brasileira de desenvolvimento e crescimento humano, 18(1), 46-52.

Pereira, T. T. S. O. (2013). Pichon-Rivière, a dialética e os grupos operativos: implicações para pesquisa e intervenção. Revista da SPAGESP, 14(1), 21-29.

Pichon-Rivière (1998). O processo grupal. São Paulo: Martins Fontes.

Rena, F., \& Mirian, P. (1998). Intervention with groups of parents of preschool children with hearing loss. Volta Review, 100(1), 1927.

Ribeiro, S. F. R., Yamada, M. O., \& Silva, C. (2005) Grupo de acompanhantes de pacientes com implante coclear: uma ação interdisciplinar da psicologia e do serviço social. Revista da SPAGESP, 6(1), 48-56.

Ribeiro, S. F. R., Yamada, M. O., \& Tavano, L. D. (2007). Vivência de mães de crianças com deficiência auditiva em sala de espera. Psicologia em Revista, 13(1), 91-106.

Silva, N. M., Paiva, F. C., \& Silveira, A. A. B. (2012) Grupoterapia e deficiência auditiva infantil: trabalhando com mães e crianças. Revista da SPAGESP, 13(2), 56-67.

Souza, L. V., Santos, M. A., Moura, F. E. G. A., Campos-Brustelo, T. N., \& Savioli, C. M. G. (2010). Fatores terapêuticos em um grupo de apoio multifamiliar no tratamento da anorexia e bulimia. Revista da SPAGESP, 11(2), 41-50.

Spanh C., Richter, B., Zschocke, I., Löhle, E., \& Wirsching M. (2001). The need for psychosocial support in parents with cochlear implanted children. International Journal of Pediatric Otorhinolaryngology, 45-53.

Yucel, E., Derim, D., \& Celik, D. (2008). The needs of hearing impaired children's parents who attend to auditory verbal therapy-counseling program. International Journal of Pediatric Otorhinolaryngology, 72, 1097-1111.

\section{Endereço para correspondência \\ Fernanda Pádua Rezende}

Hospital de Reabilitação de Anomalias Craniofaciais

Rua Silvio Merchione, 3-20, CEP 17012-900, Bauru - SP, Brasil

Endereço eletrônico: fernandarezende@ymail.com

Marina Morettin

Hospital de Reabilitação de Anomalias Craniofaciais

Rua Silvio Merchione, 3-20, CEP 17012-900, Bauru - SP, Brasil

Endereço eletrônico: mmorettin@gmail.com ou mmorettim@usp.br 


\section{Midori Otake Yamada}

Hospital de Reabilitação de Anomalias Craniofaciais

Rua Silvio Merchione, 3-20, CEP 17012-900, Bauru - SP, Brasil

Endereço eletrônico: midoriotake@yahoo.com.br ou miotake@centrinho.usp.br

Recebido em: 23/03/2015

Reformulado: 02/06/2016

Aceito para publicação: 13/06/2016

\section{Notas}

* Psicóloga, Mestre.

** Fonoaudióloga (Doutora) no Hospital de Reabilitação de Anomalias Crânio Faciais (HRAC-Centrinho), da Universidade de São Paulo, Bauru-SP (USP), Brasil.

*** Psicóloga (Doutora) no Hospital de Reabilitação de Anomalias Crânio Faciais (HRAC-Centrinho), da Universidade de São Paulo, Bauru-SP (USP), Brasil. 